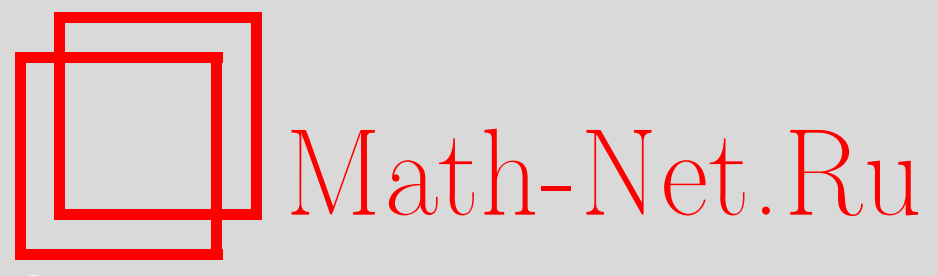

А. Н. Тимашёв, О перманентах случайных дважды стохастических матриц и асимптотических оценках чисел латинских прямоугольников и латинских квадратов, Дискрет. матем., 2002, том 14, выпуск 4, 65-86

DOI: https://doi.org/10.4213/dm264

Использование Общероссийского математического портала Math-Net.Ru подразумевает, что вы прочитали и согласны с пользовательским соглашением http://www.mathnet.ru/rus/agreement

Параметры загрузки:

IP : 34.227 .88 .159

26 апреля 2023 г., 14:56:14

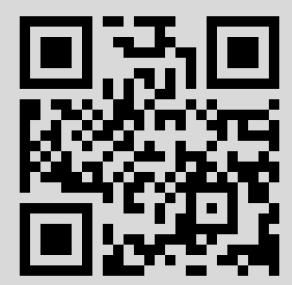




\title{
О перманентах случайных дважды стохастических матриц и асимптотических оценках чисел латинских прямоугольников и латинских квадратов
}

(ㄷ) 2002 г. А. Н. Тимашёв

\begin{abstract}
Рассматривается класс $\mathfrak{A}_{n}(k)$ всех $(0,1)$-матриц $A_{k}$ размера $n \times n$, имеющих в каждой строке и каждом столбце ровно $k$ единиц, $k=1, \ldots, n$. Доказывается асимптотическая формула для per $A_{k}$, справедливая при $n \rightarrow \infty$ и $0<n-k=o(n / \ln n)$ равномерно относительно $A_{k} \in \mathfrak{U}_{n}(k)$. Обсуждаются известные оценки сверху и снизу для чисел латинских прямоугольников размера $m \times n$ и латинских квадратов размера $n \times n$, в том числе их асимптотические выражения при $n \rightarrow \infty$ и $m=m(n)$. Отмечается, что известная гипотеза О'Нейла об асимптотике числа латинских квадратов справедлива в усиленной форме. Формулируются новые гипотезы такого типа, из которых выводятся асимптотические оценки для чисел латинских прямоугольников и латинских квадратов, улучшающие ранее известные результаты. В заключение приводится краткий обзор литературы по затронутым в статье темам с формулировками основных результатов.
\end{abstract}

Рассмотрим класс $\mathfrak{U}_{n}(k)$ всех $(0,1)$ матриц размера $n \times n$, имеющих в каждой строке и каждом столбце ровно $k$ единиц, $k=1, \ldots, n$. Если $A_{k} \in \mathfrak{A}_{n}(k)$ и per $A_{k}$ - перманент матрищы $A_{k}$, то (см. [1], с. 140; [2])

$$
n !(k / n)^{n} \leqslant \operatorname{per} A_{k} \leqslant(k !)^{n / k}, \quad k=1, \ldots, n .
$$

Нижняя оценка в (1) следует из гипотезы Ван-дер-Вардена, доказанной в [3], если каждый элемент матрицы $A_{k}$ разделить на $k$; при этом получится дважды стохастическая матрица размера $n \times n$, перманент которой не меньше, чем $n ! n^{-n}$. Верхняя оценка в (1) следует из гипотезы X. Минка (см. [1], с. 151]), доказанной в [2].

Известно (см. [1], с. 140]), что если $L(n, m)$ - число латинских прямоугольников, состоящих из $m$ строк и $n$ столбцов, элементами которых являются числа $1, \ldots, n$, то

$$
\frac{(n !)^{m+n}}{n^{m n}((n-m) !)^{n}} \leqslant L(n, m) \leqslant \prod_{k=n-m+1}^{n}(k !)^{(n / k)}, \quad m=1, \ldots, n .
$$

В частности, $L(n, n)=L_{n}-$ число латинских квадратов размера $n \times n$. В этом случае из

3 Дискретная математика, т.14 №4 
(2) следует, что

$$
\frac{(n !)^{2 n}}{n^{n^{2}}} \leqslant L_{n} \leqslant \prod_{k=1}^{n}(k !)^{n / k}
$$

Заметим, что при $n \rightarrow \infty$

$$
\frac{(n !)^{2 n}}{n^{n^{2}}}=\left(n / e^{2}\right)^{n^{2}}(2 \pi n)^{n} e^{1 / 6}(1+O(1 / n)) .
$$

Используя формулу Стирлинга, нетрудно убедиться в том, что при $\boldsymbol{n} \rightarrow \infty$

$$
\prod_{k=1}^{n}(k !)^{n / k}=\left(n / e^{2}\right)^{n^{2}} \exp \left((n / 4) \ln ^{2} n(1+O(1 / \ln n))\right) .
$$

С учетом (3) получаем, что

$$
\left(n / e^{2}\right)^{n^{2}}(2 \pi n)^{n} e^{1 / 6}(1+O(1 / n)) \leqslant L_{n} \leqslant\left(n / e^{2}\right)^{n^{2}} \exp \left((n / 4) \ln ^{2} n(1+O(1 / \ln n))\right) .
$$

В работе О’Нейла [4] была высказана гипотеза о том, что при $n \rightarrow \infty$

$$
L_{n}^{1 / n^{2}} \sim n / e^{2}
$$

Из (4) следует, что гипотеза (5) справедлива в усиленной форме: при $n \rightarrow \infty$

$$
L_{n}^{1 / n^{2}}=\left(n / e^{2}\right)\left(1+O\left(\ln ^{2} n / n\right)\right) .
$$

Более того, если при $n \rightarrow \infty$ функция $f(n) \rightarrow \infty$ так, что $\ln ^{2} n=o(f(n))$, то

$$
L_{n}^{1 / n f(n)}=\left(\frac{n}{e^{2}}\right)^{n / f(n)}\left(1+O\left(\frac{\ln ^{2} n}{f(n)}\right)\right)
$$

(в частности, при $f(n)=n, n=1,2, \ldots$, отсюда следует (6)).

Теорема 1. Пусть $n \rightarrow \infty u k=k(n) \rightarrow \infty$ так, что $0<n-k=o(n / \ln n)$. Тогда равномерно относительно $A_{k} \in \mathfrak{A}_{n}(k)$

$$
\text { per } A_{k}=\sqrt{2 \pi n}\left(\frac{k}{e}\right)^{n}\left(1+O\left(\frac{(n-k) \ln n}{n}\right)\right) .
$$

Доказательство. Утверждение теоремы 1 означает, что последовательность

$$
t_{n}=\frac{n}{(n-k) \ln n} \max _{A_{k} \in \mathfrak{A}_{n}(k)}\left|(2 \pi n)^{-1 / 2}(e / k)^{n} \operatorname{per} A_{k}-1\right|, \quad n=2,3, \ldots,
$$

ограничена.

Поскольку при $n \rightarrow \infty$

$$
n !(k / n)^{n}=\sqrt{2 \pi n}(k / e)^{n}(1+O(1 / n)),
$$

с учетом (1) достаточно убедиться в том, что

$$
(k !)^{n / k}=\sqrt{2 \pi n}(k / e)^{n}(1+O((n-k) \ln n / n)) .
$$


В условиях теоремы $k \sim n$, поэтому согласно формуле Стирлинга

$$
(k !)^{n / k}=(2 \pi k)^{n / 2 k}(k / e)^{n}(1+O(1 / n)) .
$$

Таким образом, достаточно проверить, что

$$
(2 \pi k)^{n / 2 k}=\sqrt{2 \pi n}\left(1+O\left(\frac{(n-k) \ln n}{n}\right)\right) .
$$

Логарифмируя это равенство, получаем, что

$$
\frac{n-k}{2 k} \ln (2 \pi)+\frac{n \ln k-k \ln n}{2 k}=O\left(\frac{(n-k) \ln n}{n}\right) .
$$

Так как

$$
\frac{n-k}{2 k} \ln (2 \pi)=O((n-k) / k)
$$

нужно показать, что

$$
n \ln k-k \ln n=O((n-k) \ln n) .
$$

Пусть $g_{n}=n-k=o(n / \ln n)>0$, тогда

$$
\frac{n \ln k-k \ln n}{(n-k) \ln n}=1+\frac{n \ln \left(1-g_{n} / n\right)}{g_{n} \ln n}
$$

и при $n \rightarrow \infty$

$$
\frac{n \ln \left(1-g_{n} / n\right)}{g_{n} \ln n}=-\frac{1+o(1)}{\ln n}=o(1)
$$

откуда следует (7).

Теорема 1 доказана.

Замечание 1. Тем же методом несложно показать, что

$$
\prod_{k=n-m+1}^{n}(k !)^{n / k}=\frac{(n !)^{m+n}}{n^{m n}((n-m) !)^{n}}(1+o(1))
$$

при $n \rightarrow \infty$ и $m=o(\sqrt{n / \ln n})$. Следовательно, при таких условиях согласно (2)

$$
L(n, m)=(n !)^{m} \exp \left(-\left(\begin{array}{l}
m \\
2
\end{array}\right)-\frac{m^{3}}{6 n}\right)(1+o(1)) .
$$

Известно [5], что оценка (8) на самом деле справедлива при $m=o(\sqrt{n})$. Более сильный результат установлен в [6], где, в частности, получена асимптотика числа латинских прямоугольников $L(n, m)$ при

$$
m=O\left(n^{1-\delta}\right)
$$

где $\delta \in(1 / 7,1)-$ постоянная.

Поскольку $L(n, n-1)=L(n, n)$, для латинских квадратов можно положить $m=n-1$, и тогда условие (9) не выполняется. Тем не менее, при небольших значениях $n$ наблюдается достаточно хорошее согласие между оценкой $\tilde{L}_{n}$ из статьи [6] при $m=n-1$ и статистическими оценками чисел $L_{n}$ (см. [7]). 
Замечание 2. Из неравенств (1) следует, что при $k \rightarrow \infty$ равномерно относительно $A_{k} \in \mathfrak{U}_{n}(k)$

$$
\left(\operatorname{per} A_{k}\right)^{1 / n}=\frac{k}{e}\left(1+O\left(\frac{\ln k}{k}\right)\right) .
$$

Далее будем считать, что матрица $A_{k}$ выбрана случайно равновероятно из класса $\mathfrak{U}_{n}(k)$.

Соотношение (10) подтверждает еще одну гипотезу из [4], состоящую в том, что

$$
\left(\operatorname{per} A_{k}\right)^{1 / n} \sim k / e
$$

при $k \rightarrow \infty$ для почти всех матриц $A_{k} \in \mathfrak{U}_{n}(k)$. В [4] показано, что это верно при $k \rightarrow \infty$ и $k \leqslant(\ln n)^{1 / 4-\varepsilon}$, где $\varepsilon-$ постоянная, $0<\varepsilon<1 / 4$.

С другой стороны, еще в [8] была установлена справедливость этого утверждения при $n \rightarrow \infty$ и $n-k<n^{1 / 3-\eta}$, где $\eta-$ постоянная, $0<\eta<1 / 3$.

Замечание 3. Согласно (1)

$$
n !(k / n)^{n} \leqslant \mathbf{E}\left(\operatorname{per} A_{k}\right) \leqslant(k !)^{n / k}, \quad k=1, \ldots, n .
$$

В частности, при условиях теоремы 1

$$
\mathrm{E}\left(\operatorname{per} A_{k}\right)=\sqrt{2 \pi n}\left(\frac{k}{e}\right)^{n}\left(1+O\left(\frac{(n-k) \ln n}{n}\right)\right) .
$$

Справедливость (5) с учетом (11) следует из более сильной гипотезы, состоящей в том, что при $n \rightarrow \infty$

$$
L_{n}^{1 / n}=\left(\prod_{k=1}^{n} \mathrm{E}\left(\operatorname{per} A_{k}\right)\right)^{1 / n}(1+o(1))
$$

Используя (3), (7), (11), (12), можно получить оценки чисел $L_{n}$, лучшие, чем (3). Точнее, справедливо следующее утверждение.

Предложение 1. Если (12) выполняется, то для $\delta \in(0,1)$ и для всех достаточно больших значений $n$

$$
\frac{n !^{2 n}}{n^{n^{2}}} \exp \left(\frac{n(1-\delta)}{8} \ln \ln n\right)<L_{n}<\prod_{k=1}^{n}(k !)^{n / k} \exp \left(-\frac{n(1-\delta)}{64}(\ln \ln n)^{2}\right) .
$$

Доказательство. Пусть

$$
\varepsilon \in(0,1 / 4), \quad m=\left[(\ln n)^{1 / 4-\varepsilon}\right], \quad \delta \in(0,1) .
$$

В [4] показано, что при $n \rightarrow \infty$ равномерно относительно $k \in \mathbf{N}, 2 \leqslant k \leqslant m$, для математического ожидания $\mathrm{E}$ (per $A_{k}$ ) перманента матрицы $A_{k}$ справедлива формула

$$
\mathbf{E}\left(\operatorname{per} A_{k}\right)=\sqrt{\frac{2 \pi n(k-1)}{e k}}\left(\frac{(k-1)^{k-1}}{k^{k-2}}\right)^{n}\left(1+o\left(n^{-1+\delta}\right)\right)
$$


Если

$$
\gamma_{k}=\frac{\mathrm{E}\left(\operatorname{per} A_{k}\right)}{n !(k / n)^{n}}, \quad k=1, \ldots, n
$$

то согласно (11)

$$
\gamma_{k} \geqslant 1, \quad k=1, \ldots, n
$$

Далее, согласно (12)

$$
L_{n}=\exp (o(n)) \frac{(n !)^{2 n}}{n^{n^{2}}} \prod_{k=1}^{n} \gamma_{k} \geqslant \exp (o(n)) \frac{(n !)^{2 n}}{n^{n^{2}}} \prod_{k=1}^{m} \gamma_{k}
$$

причем из (14) и формулы Стирлинга следует, что

$$
\prod_{k=1}^{m} \gamma_{k}=\frac{(2 \pi m)^{(n-1) / 2}}{\sqrt{n}} \exp \left(-\frac{m-1}{2}+\frac{n \theta_{m}}{12 m}\right)(1+o(1)),
$$

где

$$
0<\theta_{m}<1, \quad \lim _{m \rightarrow \infty} \theta_{m}=1 \text {. }
$$

При $n \rightarrow \infty$

$$
\ln m=(1 / 4-\varepsilon) \ln \ln n+O\left((\ln n)^{\varepsilon-1 / 4}\right),
$$

поэтому

$$
\begin{aligned}
\sum_{k=1}^{m} \ln \gamma_{k} & =((n-1) / 2) \ln (2 \pi m)-(m-1) / 2+O\left(n(\ln n)^{\varepsilon-1 / 4}\right) \\
& =(n(1-4 \varepsilon) / 8) \ln \ln n(1+O(1 / \ln \ln n)) \\
& >(n(1-\delta) / 8) \ln \ln n
\end{aligned}
$$

при всех достаточно больших значениях $n$, если выбрать $\delta \in(4 \varepsilon, 1)$. В силу произвольности $\varepsilon \in(0,1 / 4)$ это означает, что $\delta \in(0,1)$.

Тем самым нижняя оценка в (13) установлена. Для обоснования верхней оценки заметим, что если

$$
\tilde{\gamma}_{k}=\frac{\mathbf{E}\left(\operatorname{per} A_{k}\right)}{(k !)^{n / k}}, \quad k=1, \ldots, n
$$

то из (11) следует, что

$$
\tilde{\gamma}_{k} \leqslant 1, \quad k=1, \ldots, n,
$$

и поэтому согласно (12)

$$
L_{n}=\exp (o(n)) \prod_{k=1}^{n}\left((k !)^{n / k} \tilde{\gamma}_{k}\right) \leqslant \exp (o(n)) \prod_{k=1}^{n}(k !)^{n / k} \prod_{k=1}^{m} \tilde{\gamma}_{k},
$$

причем из (14) следует, что

$$
\prod_{k=1}^{m} \tilde{\gamma}_{k}=(2 \pi)^{(n+m-1) / 2} n^{(m-1) / 2} m^{(n-1) / 2} \exp \left(-\frac{n \ln ^{2} m}{4}(1+O(1 / \ln m))\right)(1+o(1)) .
$$


Таким образом,

$$
\begin{aligned}
\sum_{k=1}^{m} \ln \tilde{\gamma}_{k} & =-(n / 4) \ln ^{2} m(1+O(1 / \ln m)) \\
& =-(n / 4)(1 / 4-\varepsilon)^{2}(\ln \ln n)^{2}(1+O(1 / \ln \ln n)) \\
& <-(n / 64)(1-\delta)(\ln \ln n)^{2}
\end{aligned}
$$

при всех достаточно больших значениях $n$, если выбрать $\delta \in(4 \varepsilon(2-4 \varepsilon), 1)$. В силу произвольности $\varepsilon \in(0,1 / 4)$ это означает, что $\delta \in(0,1)$, откуда следует верхняя оценка в (13).

Предложение 1 доказано.

Замечание 4. Упомянутая в замечании 1 оценка $\tilde{L}_{n}$ из $[6]$ при любом $\delta \in(0,1)$ имеет вид

$$
\tilde{L}_{n}=\frac{(n !)^{2 n}}{n^{n^{2}}} \exp (O(n)) .
$$

Если гипотеза (12) справедлива и $L_{n}^{*}-$ нижняя оценка чисел $L_{n}$ в (13), то при всех достаточно больших значениях $n$

$$
\tilde{L}_{n}<L_{n}^{*}<L_{n}
$$

Более того, для любого фиксированного значения $\delta \in(0,1)$

$$
\tilde{L}_{n}=o\left(L_{n}^{*}\right)
$$

при $n \rightarrow \infty$, и поэтому $\tilde{L}_{n}=o\left(L_{n}\right)$, так что при сделанном предположении оценка $\tilde{L}_{n}$ имеет относительную погрешность, сколь угодно близкую к 1 при всех достаточно больших значениях $n$.

Замечание 5. Используя (7), нетрудно проверить, что при условиях теоремы 1 оценка (14) остается справедливой, если остаточный член вида $o\left(n^{-1+\delta}\right)$ заменить на $O((n-k) / n)$. С другой стороны, полагая

$$
f_{n}(k)=\sqrt{2 \pi n(k-1) /(e k)}\left((k-1)^{k-1} / k^{k-2}\right)^{n}, \quad k=2, \ldots, n,
$$

можно показать, что при $k \rightarrow \infty$

$$
n !(k / n)^{n}(1+o(1)) \leqslant f_{n}(k) \leqslant(k !)^{n / k}(1+o(1)) .
$$

При ограниченных значениях $k$ и $n \rightarrow \infty$ (16) следует из (11) и (14).

Изложенные соображения позволяют сформулировать еще одну гипотезу, состоящую в том, что при $n \rightarrow \infty$ и $2 \leqslant k \leqslant n$ для математического ожидания $\mathrm{E}\left(\right.$ per $A_{k}$ ) перманента случайной матрицы $A_{k}$ справедлива формула (14) с заменой остаточного члена вида $o\left(n^{-1+\delta}\right)$ на $o(1)$.

Замечание 6. При $n \rightarrow \infty$

$$
\prod_{k=2}^{n} f_{n}(k)=\frac{e^{2 / 3}}{\sqrt{2 \pi}} L_{0}(n)(1+O(1 / n))
$$


Таблица 1.

\begin{tabular}{cccccccccccc}
\hline$n$ & 4 & 5 & 6 & 7 & 8 & 9 & 10 & 11 & 12 & 13 & 14 \\
\hline$\beta_{n}$ & 1,2419 & 1,0096 & 1,1477 & 1,1233 & 1,1311 & 1,1356 & 1,1400 & 1,14 & 1,14 & 1,15 & 1,15 \\
\hline
\end{tabular}

\section{Таблица 2.}

\begin{tabular}{ccccc}
\hline$n$ & $L_{n}$ & $L_{0}(n)$ & $(\pi / e) L_{0}(n)$ & $\varepsilon_{n}$ \\
\hline 4 & 576 & 464 & 536 & $-0,069$ \\
5 & 161280 & 159751 & 184629 & 0,145 \\
6 & $8,129 \cdot 10^{8}$ & $7,083 \cdot 10^{8}$ & $8,185 \cdot 10^{8}$ & 0,007 \\
7 & $6,148 \cdot 10^{13}$ & $5,473 \cdot 10^{13}$ & $6,325 \cdot 10^{13}$ & 0,029 \\
8 & $1,088 \cdot 10^{20}$ & $0,962 \cdot 10^{20}$ & $1,111 \cdot 10^{20}$ & 0,022 \\
9 & $5,525 \cdot 10^{27}$ & $4,865 \cdot 10^{27}$ & $5,622 \cdot 10^{27}$ & 0,018 \\
10 & $9,982 \cdot 10^{36}$ & $8,756 \cdot 10^{36}$ & $1,012 \cdot 10^{37}$ & 0,014 \\
11 & $7,76 \cdot 10^{47}$ & $6,79 \cdot 10^{47}$ & $7,85 \cdot 10^{47}$ & 0,012 \\
12 & $3,10 \cdot 10^{60}$ & $2,71 \cdot 10^{60}$ & $3,13 \cdot 10^{60}$ & 0,010 \\
13 & $7,47 \cdot 10^{74}$ & $6,52 \cdot 10^{74}$ & $7,54 \cdot 10^{74}$ & 0,009 \\
14 & $1,26 \cdot 10^{91}$ & $1,10 \cdot 10^{91}$ & $1,27 \cdot 10^{91}$ & 0,008 \\
\hline
\end{tabular}

где

$$
L_{0}(n)=\left(n / e^{2}\right)^{n^{2}} n^{(3 n-2) / 2}\left(8 \pi^{3} / e\right)^{n / 2}
$$

Если положить

$$
L_{n}=L_{0}(n) \beta_{n}, \quad n=1,2, \ldots,
$$

то окажется, что при небольших значениях $n$ числа $\beta_{n}$ близки к 1 . Приведем таблицу этих чисел при $4 \leqslant n \leqslant 14$ (точные значения $L_{n}$ при $n \leqslant 9$ заимствованы из [9], а при $n=10$ из [10], откуда взяты также оценки $L_{n}$ при $\left.11 \leqslant n \leqslant 14\right)$. Вычисления велись с точностью до $10^{-4}$ при $n \leqslant 10$ и $10^{-2}$ при $11 \leqslant n \leqslant 14$.

Из таблицы 1 видно, что числа $L_{0}(n)$ достаточно хорошо аппроксимируют значения $L_{n}$ (по крайней мере, при небольших значениях $n$ ). Нетрудно также проверить, что при $n \rightarrow \infty$

$$
\tilde{L}_{n}=o\left(L_{0}(n)\right)
$$

(см. замечание 4).

Исходя из приведенных результатов, можно сформулировать гипотезу, состоящую в том, что при $n \rightarrow \infty$ существует предел

$$
\lim _{n \rightarrow \infty} \beta_{n}=\pi / e=1,1557273 \ldots,
$$

что равносильно соотношению

$$
L_{n}=(\pi / e) L_{0}(n)(1+o(1)) .
$$

При этом величины $L_{0}(n)$ определяются согласно (17). Приведем таблицу значений $L_{n}, L_{0}(n),(\pi / e) L_{0}(n)$ и $\varepsilon_{n}=\pi L_{0}(n) /\left(e L_{n}\right)-1$ при $4 \leqslant n \leqslant 14$.

Числовые данные, приведенные в таблице 2, позволяют предположить, что гипотеза (18), по-видимому, справедлива. Сформулируем несколько следствий (18). 
Следствие 1. При $n \rightarrow \infty$

$$
\frac{L_{n+1}}{L_{n}}=\sqrt{8 \pi^{3} e}\left(\frac{n^{2}}{e^{3}}\right)^{n} n^{5 / 2}(1+o(1)) .
$$

Нетрудно проверить, что относительная погрешность оценки (19) (с отбрасыванием остаточного члена $o(1))$ при $n=9$ равна $-0,015$, а при $n=13$ равна $-0,006$ (с точностью до $10^{-3}$ ).

Следствие 2. При $n \rightarrow \infty$

$$
L_{n}^{1 / n}=\sqrt{\frac{8 \pi^{3}}{e}}\left(\frac{n}{e^{2}}\right)^{n} n^{3 / 2}\left(1-\frac{\ln n}{n}+\frac{\ln \pi-1}{n}+o\left(\frac{1}{n}\right)\right) .
$$

При $n=13$ оценка (20) обладает относительной погрешностью, примерно равной $-0,019$.

Следствие 3. При $n \rightarrow \infty$

$$
\begin{aligned}
L_{n}^{1 / n^{2}}=\frac{n}{e^{2}}\left(1+\frac{3 \ln n}{2 n}+\frac{\ln C}{n}\right. & +\frac{9 \ln ^{2} n}{8 n^{2}} \\
& \left.+\frac{(3 \ln C-2) \ln n}{2 n^{2}}+\frac{\ln ^{2} C+2 \ln \pi-2}{2 n^{2}}+o\left(\frac{1}{n^{2}}\right)\right),
\end{aligned}
$$

где $C=\sqrt{8 \pi^{3} / e}=9,55262131 \ldots, \ln C=2,2568156 \ldots$

В свою очередь, оценка (21) при $n=13$ дает относительную погрешность порядка $-0,007$.

Следствие 4. Пусть $l_{n}-$ число редучированных латинских квадратов порядка $n$ (y каждого из таких квадратов первая строка и первый столбеч совпадают с вектором $(1, \ldots, n))$. Тогда при $n \rightarrow \infty$

$$
l_{n}=\frac{1}{2 e} l_{0}(n)(1+o(1)),
$$

гдe

$$
l_{0}(n)=\left(n / e^{2}\right)^{n^{2}} n^{-(n+2) / 2}\left(8 \pi^{3} e^{3}\right)^{n / 2} .
$$

Утверждение следствия 4 с учетом (17), (18) следует из формулы

$$
L_{n}=n !(n-1) ! l_{n}
$$

и формулы Стирлинга.

Ниже приведена таблища значений $l_{n}, l_{0}(n)$ и $\delta_{n}=l_{0}(n) /\left(2 e l_{n}\right)-1$ для $4 \leqslant n \leqslant 15$.

При $n=16$ методом экстраполяции в [10] получена заниженная оценка $l_{16} \cong 10^{102}$, на самом деле $l_{16} \cong(1 / 2 e) l_{0}(16) \cong 7,9 \cdot 10^{103}$. 
Таблица 3.

\begin{tabular}{cccr}
\hline$n$ & $(1 / 2 e) l_{0}(n)$ & $l_{n}$ & $\delta_{n}$ \\
\hline 4 & 3,88 & 4 & $-0,030$ \\
5 & 66 & 56 & 0,179 \\
6 & 9741 & 9408 & 0,035 \\
7 & $1,785 \cdot 10^{7}$ & $1,694 \cdot 10^{7}$ & 0.054 \\
8 & $5,584 \cdot 10^{11}$ & $5,353 \cdot 10^{11}$ & 0,043 \\
9 & $3,915 \cdot 10^{17}$ & $3,776 \cdot 10^{17}$ & 0,037 \\
10 & $7,814 \cdot 10^{24}$ & $7,581 \cdot 10^{24}$ & 0,031 \\
11 & $5,50 \cdot 10^{33}$ & $5,36 \cdot 10^{33}$ & 0,026 \\
12 & $1,66 \cdot 10^{44}$ & $1,62 \cdot 10^{44}$ & 0,025 \\
13 & $2,56 \cdot 10^{56}$ & $2,51 \cdot 10^{56}$ & 0,020 \\
14 & $2,37 \cdot 10^{70}$ & $2,33 \cdot 10^{70}$ & 0,017 \\
15 & $1,54 \cdot 10^{86}$ & $1,50 \cdot 10^{86}$ & \\
\hline
\end{tabular}

Замечание 7. Аналогичный подход можно использовать для асимптотической оценки числа латинских прямоугольников при $n \rightarrow \infty$ и $1 \leqslant m=m(n) \leqslant n-1$.

Из (15) следует, что

$$
\prod_{k=n-m+1}^{n} f_{n}(k)=(2 \pi n / e)^{m / 2}(1-m / n)^{n^{2}-m n+1 / 2} \frac{(n !)^{2 n}}{n^{m n}((n-m) !)^{2 n}} .
$$

Полагая при $n \rightarrow \infty$

$$
L(n, m)=C(n, m) \prod_{k=n-m+1}^{n} f_{n}(k)(1+o(1))
$$

из (18) и формулы

$$
\prod_{k=2}^{n} f_{n}(k)=\frac{e^{2 / 3} L_{n}}{\sqrt{2 \pi} \beta_{n}}(1+O(1 / n))
$$

при $m=n-1$ получаем, что

$$
L_{n}=L(n, n-1)=\sqrt{2 \pi^{3}} e^{-5 / 3} \prod_{k=2}^{n} f_{n}(k)(1+o(1)) .
$$

Учитывая (11), (14), (16), (22), (23), (24), можно предположить, что при $n \rightarrow \infty$ и $1 \leqslant m=m(n) \leqslant n-1$

$$
L(n, m)=C(n, m)(2 \pi n / e)^{m / 2}(1-m / n)^{n^{2}-m n+1 / 2}(n ! /(n-m) !)^{2 n} n^{-m n}(1+o(1)),
$$

где

$$
1 \leqslant C(n, m) \leqslant \sqrt{2 \pi^{3}} e^{-5 / 3}=1,487358536 \ldots
$$

Если $m=n-1$, то согласно (23), (24) можно положить

$$
C(n, n-1)=\sqrt{2 \pi^{3}} e^{-5 / 3} \text {. }
$$


Если $n \rightarrow \infty$ и $m=o(\sqrt{n})$, то нетрудно проверить, что правая часть равенства (25) (без учета множителя $C(n, m))$ равна

$$
(n !)^{m} \exp \left(-\left(\begin{array}{c}
m \\
2
\end{array}\right)-\frac{m^{3}}{6 n}\right)(1+o(1)),
$$

что совпадает с известной асимптотической оценкой чисел $L(n, m)$ (см. [5]). Таким образом, при $m=o(\sqrt{n})$ можно положить $C(n, m)=1$, и тогда оценка (25) будет выполняться.

Используя формулу Стирлинга

$$
n !=\sqrt{2 \pi n}(n / e)^{n} \exp \left(\theta_{n} /(12 n)\right)
$$

где $0<\theta_{n}<1$ и $\lim _{n \rightarrow \infty} \theta_{n}=1$, нетрудно убедиться в том, что правая часть (25) может быть записана в виде

$$
\begin{aligned}
C(n, m) \frac{(n !)^{m+n}}{n^{m n}((n-m) !)^{n}} & (1-m / n)^{-(n-1) / 2} \\
& \times \exp \left(-m / 2+(1 / 12)\left(1-m / n-n \theta_{n-m} /(n-m)\right)\right)(1+o(1)) .
\end{aligned}
$$

Следовательно, если $n \rightarrow \infty$ и $m=o(n)$, то правая часть (25) без учета множителя $C(n, m)$ равна

$$
\frac{(n !)^{n+m}}{n^{m n}((n-m) !)^{n}}(1-m / n)^{-n / 2} e^{-m / 2}(1+o(1)),
$$

что совпадает с асимптотической оценкой чисел $L(n, m)$ из [6]. Эта оценка доказана в [6] при условии, что $m=o\left(n^{6 / 7}\right)$, и там же высказана гипотеза, согласно которой результат остается верным, если при $n \rightarrow \infty$ величина $m=O\left(n^{1-\delta}\right)$, где $\delta$ фиксировано и $0<\delta \leqslant 1$.

Исходя из сказанного выше, можно предположить, что то же верно и при $m=o(n)$. Если это предположение справедливо, то при $m=o(n)$ в (25) можно считать, что $C(n, m)=1$.

Изложенные соображения косвенным образом подтверждают справедливость оценок (25), (26) при $n \rightarrow \infty$ и $1 \leqslant m=m(n) \leqslant n-1$. Численные расчеты показывают, что величины $C(n, m)$ действительно заключены в границах (26). Если, например, $n=10$ и $m=3$, то $L(10 ; 3) \cong 2,131 \cdot 10^{18}$ (это значение заимствовано из [11]), и вычисления по формуле (25) дают $C(10 ; 3) \cong 1,139$; если же $n=13$, то $L(13 ; 3) \cong 1,106 \cdot 10^{28}$, и поэтому $C(13 ; 3) \cong 1,104$.

В [6] сформулирована еще одна гипотеза, состоящая в том, что существуют постоянные $C_{1}$ и $C_{2}$ такие, что $0 \leqslant C_{1} \leqslant C_{2}$ и при $1 \leqslant m \leqslant n$

$$
\exp \left(C_{1} m^{2} / n\right) \leqslant L(n, m)(n !)^{-m}\left(\frac{n(n-1) \ldots(n-m+1)}{n^{m}}\right)^{-n} \leqslant \exp \left(C_{2} m^{2} / n\right)
$$

Однако при $m=n-1$ из (17), (18) и формулы Стирлинга следует, что

$$
\ln \left(L_{n}(n !)^{-2 n+1} n^{n^{2}-n}\right)=\frac{1}{2} n \ln n(1+o(1)),
$$

так что верхняя оценка в (27) при больших $n$ не может выполняться. То же утверждение можно вывести из нижней оценки (13). 
Получить оценки величин $C(n, m)$, более точные, чем (26), пока не удалось. Заметим, однако, что если положить

$$
\tilde{C}(n, m)=1+\left(C_{0}-1\right) m / n, \quad m=1, \ldots, n-1,
$$

где

$$
C_{0}=\sqrt{2 \pi^{3}} e^{-5 / 3}
$$

то окажется, что числа $\tilde{C}(n, m)$ достаточно хорошо аппроксимируют значения $C(n, m)$. Так, в условиях рассмотренного выше примера, когда $m=3$, при $n=10$ получаем $\tilde{C}(10 ; 3) \cong 1,146$ с относительной погрешностью порядка 0,006 ; при $n=13$ справедливо соотношение $\tilde{C}(13 ; 3) \cong 1,112$ с относительной погрешностью 0,007 .

В заключение, не претендуя на полноту, приведем краткий обзор литературы по темам, затронутым в работе, с формулировками основных результатов.

Впервые перманенты дважды стохастических матриц, по-видимому, были рассмотрены в [12], где, в частности, показано, что такие перманенты всегда положительны. Там же установлено, что если $A_{k} \in \mathfrak{A}_{n}(k)$, то per $A_{k} \geqslant k$. Гипотеза Ван-дер-Вардена сформулирована в [13]: если $A$ - дважды стохастическая матрица размера $n \times n$, то $\operatorname{per} A \geqslant n ! n^{-n}$ с равенством тогда и только тогда, когда все элементы матрицы $A$ равны $1 / n$. Как уже упоминалось выше, эта гипотеза доказана в [3] (и независимо в [14] без обоснования единственности минимизирующей матрицы). Гипотеза Минка впервые сформулирована в [15]: если $A-(0,1)$-матрица размера $n \times n$ со строчными суммами $r_{1}, \ldots, r_{n} \geqslant 1$, то

$$
\operatorname{per} A \leqslant \prod_{i=1}^{n}\left(r_{i} !\right)^{1 / r_{i}}
$$

Доказательство этого утверждения приведено в [2].

В [16] рассмотрен класс дважды стохастических матриц вида

$$
A=\frac{1}{s} \sum_{i=1}^{s} \Pi_{i}
$$

где $\Pi_{1}, \ldots, \Pi_{s}-$ подстановочные матрицы размера $n \times n$. В предположении, что на множестве всех $(n !)^{s}$ упорядоченных наборов таких матриц задано равномерное распределение, установлено, что

$$
\mathbf{E}(\operatorname{per} A)=\frac{1}{s^{n}(n !)^{s-2}} \sum_{r_{1}+\ldots+r_{s}=n} \frac{\left(n-r_{1}\right) ! \ldots\left(n-r_{s}\right) !}{r_{1} ! \ldots r_{s} !},
$$

где суммирование осуществляется по всем решениям уравнения

$$
r_{1}+\ldots+r_{s}=n
$$

в целых неотрицательных числах $r_{1}, \ldots, r_{s}$. Показано, что для любого фиксированного $n=1,2, \ldots$ имеют место равенства

$$
\lim _{s \rightarrow \infty} \mathbf{E}(\operatorname{per} A)=n ! n^{-n}
$$

В случае фиксированного $s \geqslant 3$ и $n \rightarrow \infty$ доказана формула

$$
\mathbf{E}(\operatorname{per} A)=\sqrt{2 \pi n}(1-1 / s)^{n(s-1)+s-1 / 2}(1-2 / s)^{-(s-1) / 2}(1+o(1)) .
$$


Нижняя граница перманента $(0,1)$-матрицы $A$ со строчными суммами

$$
r_{1} \geqslant \ldots \geqslant r_{n}
$$

впервые получена в [17]:

$$
\text { per } A \geqslant \prod_{i=1}^{n} \max \left\{r_{i}+i-n, 0\right\}
$$

Простое доказательство этого неравенства приведено в [18]. В [19] высказана гипотеза, состоящая в том, что если $A_{k} \in \mathfrak{A}_{n}(k), n=1,2, \ldots$, то для фиксированного $k \geqslant 3$

$$
\liminf _{n \rightarrow \infty}\left(\operatorname{per} A_{k}\right)^{1 / n}>1
$$

(справедливость этого утверждения сразу следует из (1)).

Случайные матрицы из класса $\mathfrak{A}_{n}(k)$ рассматривались в упоминавшейся выше работе [4]. Для математического ожидания $\mathrm{E}\left(\operatorname{per} A_{k}\right)$ перманента матрищы $A_{k}$, выбранной случайно равновероятно из класса $\mathfrak{U}_{n}(k)$, справедлива оценка (14).

Для второго момента $\mathbf{E}\left(\operatorname{per} A_{k}\right)^{2}$ в [4] получена асимптотическая формула

$$
\mathbf{E}\left(\operatorname{per} A_{k}\right)^{2}=\frac{2 \pi n(k-1)}{e k}\left(\frac{(k-1)^{k-1}}{k^{k-2}}\right)^{2 n}\left(1+1 / 4(k-1)^{4}+O\left(1 / k^{6}\right)\right)\left(1+O\left(n^{-1 / 2+\delta}\right)\right),
$$

где

$$
3 \leqslant k<(\ln n)^{1 / 4-\varepsilon}, \quad 0<\varepsilon<1 / 4, \quad 0<\delta<1 / 2 .
$$

Из этих результатов следует, что если $k=k(n) \in \mathbf{N}-$ монотонно возрастающая функция от $n$, не ограниченная сверху и такая, что $k(n)<(\ln n)^{1 / 4-\varepsilon}$, то при $n \rightarrow \infty$

$$
\operatorname{per} A_{k(n)} / \mathrm{E}\left(\operatorname{per} A_{k(n)}\right) \rightarrow 1
$$

по вероятности.

В статье [20] рассматривался класс $\mathfrak{U}(n, N)$ всех $(0,1)$-матриц размера $n \times n$, имеющих $N$ единиц, $n \leqslant N \leqslant n^{2}$. Если матрица $A_{n, N}$ выбрана случайно равновероятно из этого класса, то при $n \rightarrow \infty$

$$
\mathrm{E}\left(\operatorname{per} A_{n, N}\right)=n !\left(N / n^{2}\right)^{n} \exp \left(-(1 / 2)\left(n^{2} / N-1\right)\right)\left(1+O\left(1 / n^{2 \varepsilon}\right)\right)
$$

в предположении, что $N>n^{3 / 2+\varepsilon}$, где постоянная $\varepsilon$ лежит в интервале $(0,1 / 2)$. При этих же условиях

$$
\mathbf{E}\left(\operatorname{per} A_{n, N}\right)^{2}=(n !)^{2}\left(N / n^{2}\right)^{2 n} \exp \left(-\left(n^{2} / N-1\right)\right)\left(1+O\left(1 / n^{1 / 4+\varepsilon}\right)+O\left(1 / n^{2 \varepsilon}\right)\right) .
$$

Из этих результатов следует, что если $N=N(n)>n^{3 / 2+\varepsilon}$, то при $n \rightarrow \infty$

$$
\operatorname{per} A_{n} n, N(n)\left(\mathbf{E}\left(\operatorname{per} A_{n, N(n)}\right)\right)^{-1} \rightarrow 1
$$

по вероятности. Это утверждение допускает следующую модификацию, также приведенную в [20]. Пусть $\mathfrak{A}_{n}(R, S)$ - класс всех $(0,1)$-матриц размера $n \times n$, имеющих

$$
R=\left(r_{1}, \ldots, r_{n}\right), \quad S=\left(s_{1}, \ldots, s_{n}\right)
$$


в качестве векторов строчных и столбцовых сумм. В частности, если $r_{j}=s_{j}=k$, $j=1, \ldots, n$, то

$$
\mathfrak{U}_{n}(R, S)=\mathfrak{U}_{n}(k) .
$$

Класс матриц $\mathfrak{X}_{n}(R, S)$ называется разреженным с функщией $f(n)$, если

$$
r_{j}<f(n), \quad s_{j}<f(n), \quad j=1, \ldots, n .
$$

Этот же класс называется сгущенным с функщией $f(n)$, если

$$
r_{j}>n-f(n), \quad s_{j}>n-f(n), \quad j=1, \ldots, n .
$$

Пусть $A \in \mathfrak{A}_{n}(R, S)$, где $\mathfrak{U}_{n}(R, S)$ - сгущенный класс $(0,1)$-матриц с функцией

$$
f(n)=(\ln n)^{1-\varepsilon},
$$

где $\varepsilon$ - постоянная, $\varepsilon \in(0,1)$, и

$$
L(n)=n^{2}-\sum_{j=1}^{n} r_{j}
$$

- число нулей в матрице $A$.

Тогда при $n \rightarrow \infty$

$$
\text { per } A=n ! \exp (-L(n) / n)\left(1+O\left(n^{-1+\delta}\right)\right),
$$

где $\delta$ - постоянная, $\delta \in(0,1)$.

Если же $\mathfrak{Q}_{n}(R, S)$ - разреженный класс $(0,1)$-матриц с функцией

$$
f(n)=(\ln n)^{1 / 4-\varepsilon}
$$

где $\varepsilon$ - постоянная, $\varepsilon \in(0,1 / 4)$, то число матриц в таком классе при $n \rightarrow \infty$ есть

$$
\frac{\left(\sum_{j=1}^{n} r_{j}\right) !}{\prod_{j=1}^{n}\left(r_{j} ! s_{j} !\right)} \exp \left(-\frac{\sum_{j=1}^{n} r_{j}\left(r_{j}-1\right) \sum_{j=1}^{n} s_{j}\left(s_{j}-1\right)}{2\left(\sum_{j=1}^{n} r_{j}\right)^{2}}\right)\left(1+O\left(n^{-1+\delta}\right)\right)
$$

где $\delta$ - постоянная, $\delta \in(0,1)$.

Класс $\mathfrak{U}(n ; N)$ рассматривался также в [19] в предположении, что при $n \rightarrow \infty$

$$
N=N_{1}=N_{1}(n)=n \ln n+C n+o(n),
$$

где $C$ - постоянная. При этих условиях

$$
\begin{aligned}
& \mathbf{E}\left(\operatorname{per} A_{n, N_{1}}\right)=\exp (n(\ln \ln n-1+(C-1 / 2) / \ln n+o(1 / \ln n))), \\
& \mathbf{D}\left(\operatorname{per} A_{n, N_{1}}\right)=\exp (2 n \ln \ln n+O(n)) .
\end{aligned}
$$

Пусть

$$
P\left(n, N_{1}\right)=\mathbf{P}\left\{\operatorname{per} A_{n ; N_{1}}>0\right\}
$$

тогда

$$
\lim _{n \rightarrow \infty} P\left(n, N_{1}\right)=\exp \left(-2 e^{-C}\right)
$$


Если же

$$
N_{2}=N_{2}(n)=n \ln n+n \omega(n)+o(n),
$$

To

$$
\lim _{n \rightarrow \infty} P\left(n, N_{2}\right)= \begin{cases}1 & \text { при } \omega(n) \rightarrow \infty, \\ 0 & \text { при } \omega(n) \rightarrow-\infty .\end{cases}
$$

Обозначим через $v(A)$ максимальное число попарно противоречивых подстановок, соответствующих наборам из $n$ неколлинеарных единиц $(0,1)$-матрицы $A$ размера $n \times n$. Ясно, что per $A \geqslant v(A)$, а случай $v(A) \geqslant 1$ эквивалентен неравенству per $A>0$. Положим

$$
P(n, N, r)=\mathbf{P}\left\{v\left(A_{n, N}\right) \geqslant r\right\}, \quad r=1,2 \ldots
$$

При $r=1$

$$
P(n, N, 1)=\mathbf{P}\left\{\text { per } A_{n, N}>0\right\} .
$$

В [19] установлены следующие утверждения.

Если $r \in \mathbf{N}$ фиксировано и при $n \rightarrow \infty$

$$
N_{r}^{*}=n \ln n+(r-1) n \ln \ln n+C n+o(n),
$$

где $C$ - постоянная, то

$$
\lim _{n \rightarrow \infty} P\left(n, N_{r}^{*}, r\right)=\exp \left(-2 e^{-C} /(r-1) !\right) .
$$

Если же $r \in \mathbf{N}$ фиксировано и при $n \rightarrow \infty$

$$
N_{r}=n \ln n+(r-1) n \ln \ln n+n \omega(n)+o(n),
$$

где $\omega(n) \rightarrow \infty$, то

$$
\lim _{n \rightarrow \infty} P\left(n, N_{r}, r\right)=1 .
$$

Аналогичные результаты получены и для класса случайных $(0,1)$-матрищ $A(p)=\left(a_{i j}\right)$ размера $n \times n$, у которых элементы $a_{i j}$ - независимые в совокупности случайные величины такие, что

$$
\mathbf{P}\left\{a_{i j}=1\right\}=1-\mathbf{P}\left\{a_{i j}=0\right\}=p,
$$

где $p$ не зависит от индексов $i$ и $j$. Точнее, если

$$
P_{n}(p)=\mathbf{P}\{\operatorname{per} A(p)>0\},
$$

то (см. [21], с. 88) при $n \rightarrow \infty$ и $p=(\ln n+C) / n+o(1 / n)$, где $C-$ постоянная,

$$
\lim _{n \rightarrow \infty} P_{n}(p)=\exp \left(-2 e^{-C}\right) \text {. }
$$

Если же $r \in \mathbf{N}$ фиксировано и при $n \rightarrow \infty$

$$
p=\ln n / n+(r-1) \ln \ln n / n+\omega(n) / n+o(1 / n),
$$

где $\omega(n) \rightarrow \infty$, то (см. [21], с.90)

$$
\lim _{n \rightarrow \infty} \mathbf{P}\{v(A(p)) \geqslant r\}=1 .
$$


Рассматривалась также задача оценки числа $M(n)$ всех $(0,1)$-матриц размера $n \times n$ с нулевым перманентом. В [22] показано, что при $n \rightarrow \infty$

$$
M(n)=n 2^{n^{2}-n+1}(1+o(1)) .
$$

С другой стороны (см. [23]), для любого целого положительного $r$ существуют $(0,1)$-матрицы размера $n \times n$ с перманентом, равным $r$, и минимальное значение $n$ с таким свойством не превосходит $\left[\log _{2}(r-1)\right]+2, r=2,3 \ldots$

В [24] доказано следующее утверждение. Пусть

$$
r_{k}(n)=\min \left\{\operatorname{per} A_{k} / A_{k} \in \mathfrak{A}_{n}(k)\right\},
$$

тогда

$$
r_{k}(n) \leqslant k^{2 n} /\left(\begin{array}{c}
n k \\
k
\end{array}\right), \quad k=3, \ldots, n .
$$

Это утверждение следует из (1), поскольку

$$
(k !)^{n / k} \leqslant k^{2 n} /\left(\begin{array}{c}
n k \\
k
\end{array}\right), \quad 3 \leqslant k \leqslant n .
$$

Там же была высказана гипотеза, согласно которой для любого фиксированного $k=3,4, \ldots$

$$
\lim _{n \rightarrow \infty}\left(r_{k}(n)\right)^{1 / n}=\frac{(k-1)^{k-1}}{k^{k-2}}
$$

(что согласуется с гипотезой, сформулированной в замечании 5). При $k=3$ эта гипотеза справедлива, что следует из оценки, доказанной в [25]: если $A_{3} \in \mathfrak{U}_{n}(3)$, то

$$
\text { per } A_{3} \geqslant 6(4 / 3)^{n-3}, \quad n=3,4, \ldots
$$

Перманенты случайных стохастических матриц рассматривались в статье автора [26]. Пусть $\mathfrak{B}_{n}(k)$ - класс всех $(0,1)$-матриц размера $n \times n$, имеющих в каждой строке $k$ единиц, $2 \leqslant k \leqslant n$. Если $B_{k}$ - матрица, выбранная случайно равновероятно из класса $\mathfrak{B}_{n}(k)$, то

$$
\mathbf{E}\left(\operatorname{per} B_{k}\right)=n !(k / n)^{n} .
$$

Если все элементы матрицы $B_{k}$ разделить на $k$, то получится случайная стохастическая матрица $B_{k}^{\prime}$, для которой per $B_{k}^{\prime}=k^{-n} \operatorname{per} B_{k}$, так что

$$
\mathbf{E}\left(\operatorname{per} B_{k}^{\prime}\right)=n ! n^{-n}
$$

В [26] показано, что если $k=2$, то при $n \rightarrow \infty$

$$
\mathbf{E}\left(\operatorname{per} B_{k}\right)^{2}=(\pi n / e)(2 / e)^{n}(1+o(1)) ;
$$

если же $k \geqslant 3$, то при $n \rightarrow \infty$

$$
\mathbf{E}\left(\operatorname{per} B_{k}\right)^{2}=(n !)^{2} n^{-2 n}(k(k-1))^{n} \exp ((n-1) /(k-1))(1+o(1)) .
$$

Из этих результатов следует, что если $n \rightarrow \infty$ и $k=k(n) \rightarrow \infty$ так, что $\sqrt{n}=o(k(n))$, To

$$
\frac{\operatorname{per} B_{k(n)}}{n !(k / n)^{n}} \rightarrow 1
$$


по вероятности. Условие $\sqrt{n}=o(k(n))$ означает, что $n^{3 / 2}=o(N)$, где $N=n k(n)-$ число единиц в матрище $B_{k(n)}$. Это указывает на аналогию в формулировках законов больших чисел для случайной матрищы $B_{k}$ и случайной $(0,1)$-матрищы $A_{n, N}$ (см. выше).

Заметим, что класс $(0,1)$-матриц $\mathfrak{B}_{n}(k)$ рассматривался в [27], где сформулирован результат, аналогичный теореме Эрдеша-Реньи [19], оценивающей вероятность положительности перманента случайной $(0,1)$-матрищы с заданным числом единиш. Таким образом, указанная аналогия распространяется и на закон больших чисел.

В [26] рассмотрена также случайная стохастическая матрица $C_{n}$ размера $n \times n$, строки которой являются независимыми в совокупности одинаково распределенными $n$-мерными случайными величинами, имеющими симметричное распределение Дирихле с параметром $v>0$. Отметим, что в случае $v=1$ в [28] показано, что с вероятностью 1 при $n \rightarrow \infty$ имеет место сходимость

$$
S\left(C_{n}\right) \rightarrow 1-\gamma,
$$

где $S\left(C_{n}\right)$ - пропускная способность дискретного канала без памяти с матрицей переходных вероятностей $C_{n}$ и $\gamma=0,5772 \ldots$ - постоянная Эйлера.

Справедливы следующие утверждения [26]:

$$
\mathbf{E}\left(\operatorname{per} C_{n}\right)=n ! n^{-n},
$$

и если $n \rightarrow \infty$ и $v=v(n)>0$ меняется так, что $n v(n) \rightarrow \infty$, то

$$
\mathbf{E}\left(\operatorname{per} C_{n}\right)^{2}=(n !)^{2} n^{-2 n}(1-1 /(1+n \nu))^{n} \exp (1 / \nu)(1+O(1 /(n v))) .
$$

Если же $n \rightarrow \infty$ и $v=v(n)>0$ меняется так, что $n(v(n))^{2} \rightarrow \infty$, то

$$
\left(n^{n} / n !\right) \operatorname{per} C_{n} \rightarrow 1
$$

по вероятности.

Вопрос об оценке числа $(0,1)$-матриц размера $n \times m$ при некоторых ограничениях на рост координат векторов сумм строк $\left(r_{1}, \ldots, r_{n}\right)$ и векторов сумм столбцов $\left(s_{1}, \ldots, s_{m}\right)$ рассматривался в [29].

Получены следующие результаты. Пусть $M(n, m)$ - число таких матриц, тогда при $n \rightarrow \infty$

$$
M(n, m)=\frac{N !}{\prod_{i=1}^{n}\left(r_{j} !\right) \prod_{j=1}^{m}\left(s_{j} !\right)}\left(l^{-\alpha}+r_{n}^{*}\right),
$$

где

$$
N=\sum_{i=1}^{n} r_{i}=\sum_{j=1}^{m} s_{j}
$$

причем эта формула справедлива, если выполняются условия одного из следующих трех случаев:

(1) $\alpha=(1 / 2)(l-1)^{2}, r_{n}^{*}=O\left(n^{-1+\gamma^{2} / 2}\right), r_{1}=\ldots=r_{n}=s_{1}=\ldots=s_{m}=l, m=n$, $1<l \leqslant \gamma \sqrt{\ln n}, \gamma \in(0,1)$ - постоянная;

(2) $\alpha=(1 / 2)(k-1)(l-1), r_{n}^{*}=O\left(n^{\gamma-1} \ln ^{5} n\right), r_{1}=\ldots=r_{n}=k, s_{1}=\ldots=s_{m}=l>1$, $1<k \leqslant(l-1)^{-1} \gamma \ln n, \gamma \in(0,1)$ - постоянная;

(3) $\alpha=\left(2 / N^{2}\right) \sum_{i=1}^{n}\left(\begin{array}{l}r_{i} \\ 2\end{array}\right) \sum_{j=1}^{m}\left(\begin{array}{l}s_{j} \\ 2\end{array}\right), r_{n}^{*}=O\left(n^{\gamma^{4} / 4-1 / 2} \ln ^{2} n\right)$, $l=\max \left\{r_{1}, \ldots, r_{n}, s_{1}, \ldots, s_{m}\right\} \leqslant \gamma \ln ^{1 / 4} n, \gamma \in\left(0,(2 / 3)^{1 / 4}\right)-$ постоянная. 
В [30] изучался близкий к рассматриваемой проблематике вопрос о существовании системы различных представителей случайных множеств. Набор элементов $\left(x_{1}, \ldots, x_{n}\right)$ называется системой различных представителей (или трансверсалью) семейства $X_{1}, \ldots, X_{n}$ подмножеств множества $X$, если

$$
x_{i} \in X_{i}, \quad x_{i} \neq x_{j}, \quad i, j=1, \ldots, n, \quad i \neq j
$$

Пусть $\mathfrak{P}(X)$ - множество всех подмножеств $X$. На совокупности непустых подмножеств, входящих в $\mathfrak{B}(X),|X|=m$, зададим равномерное распределение и будем предполагать, что множества $X_{1}, \ldots, X_{n}$ выбираются независимо друг от друга. При этих условиях в [30] установлена справедливость следующих утверждений.

При $n \rightarrow \infty$ вероятность того, что случайный набор непустых множеств

$$
X_{1}, \ldots, X_{n} \in \mathfrak{B}(X), \quad|X|=n,
$$

обладает трансверсалью, стремится к 1 .

При $n \leqslant m$ и $m \rightarrow \infty$ с вероятностью, стремящейся к 1 , случайный набор непустых множеств

$$
X_{1}, \ldots, X_{n} \in \mathfrak{B}(X), \quad|X|=m,
$$

обладает трансверсалью.

Свойства перманентов рассматривались в некоторых монографиях и обзорных статьях. Из имеющихся в литературе на русском языке отметим следующие издания. Наиболее полный обзор приведен в [1] (см. с. 164-209), где представлена библиография за период с 1812 по 1981 год. В этой книге есть отдельная глава, содержащая изложение вопросов применения перманентов, а также список гипотез и нерешенных проблем. Имеется также обзор [31], в котором представлен достаточно полный список литературы о перманентах. В книге [32] содержится определение перманента и краткий обзор некоторых его свойств. Монография [33] содержит главу о трансверсалях и перманентах. Здесь приводятся основные свойства перманентов, даются формулы для перманентов выпуклых комбинаций двух и трех подстановочных матриц, матричная факторизация [17], общая формула Райзеpa (см. [34], с. 33) для вычисления перманентов и некоторые их оценки. В обзоре [35] рассматриваются вопросы, связанные с перманентами случайных $(0,1)$-матриц и случайных дважды стохастических матриц. Монография [36] содержит, в частности, подробное изложение вопросов, имеющих связь с теоремой Ван-дер-Вардена-Егорычева-Фаликмана, а также асимптотические методы изучения неотрицательных матриц, в том числе оценки числа примитивных $(0,1)$-матриц, исследования случайных решеток и булевых алгебр, случайных покрытий множеств, максимальной глубины некоторых классов $(0,1)$-матрищ.

Несколько в стороне от рассматриваемых проблем находится книга [37], в которой впервые систематически исследуются асимптотические свойства перманентов матриц со случайными элементами. Асимптотический анализ случайных перманентов и их производящих функщий осуществляется в схемах нормальной и пуассоновской аптроксимации выборок. Получение новых предельных результатов основано на использовании алгебраических и аналитических свойств перманентов конечномерных проекционных матриц и их производящих функций. Применение методов ортогональных и канонических разложений и композищий позволяет провести асимптотическое исследование симметрических статистик, представленных функщионалами по случайным перманентным мерам. Приводятся отдельные примеры возможного анализа случайных перманентов матриц с зависимыми элементами. 
Подсчет числа латинских прямоугольников $L(n, m)$ при $m=2$ и $m=3$ связан с классическими комбинаторными задачами: о числе беспорядков и супружеских парах. Назовем латинский прямоугольник нормализованным, если его первая строка совпадает с вектором $(1,2, \ldots, n)$. Число $K(n, m)$ нормализованных латинских прямоугольников размера $m \times n$ связано с $L(n, m)$ соотношением

$$
L(n, m)=n ! K(n, m) .
$$

При $m=2$ число беспорядков

$$
D_{n}=K(n, 2)=n ! \sum_{i=0}^{n}(-1)^{i} / i !
$$

так что $L(n, 2) \sim(n !)^{2} e^{-1}$ при $n \rightarrow \infty$. Этот результат был известен еще Эйлеру. При $m=3$

$$
K(n, 3)=\sum_{k=0}^{[n / 2]}\left(\begin{array}{l}
n \\
k
\end{array}\right) D_{n-k} D_{k} U_{n-2 k}
$$

где

$$
U_{0}=1, \quad U_{n}=\sum_{k=0}^{n}(-1)^{k} \frac{2 n}{2 n-k}\left(\begin{array}{c}
2 n-k \\
k
\end{array}\right)(n-k) !, \quad n=1,2, \ldots,
$$

- число размещений в задачах о супружеских парах, то есть трехстрочных латинских прямоугольников, первые две строки которых суть

$$
\left(\begin{array}{cccc}
1 & 2 & \ldots & n \\
n & 1 & \ldots & n-1
\end{array}\right)
$$

Формула для $U_{n}$ получена в [38]; соответствующий результат для $K(n, 3)$ доказан в [39]. При $n \rightarrow \infty$ справедливо асимптотическое разложение (см. [11], с. 264)

$$
U_{n} \sim n ! e^{-2}\left(1-\frac{1}{n-1}+\frac{1}{2 !(n-1)_{2}}-\ldots+\frac{(-1)^{k}}{k !(n-1)_{k}}+\ldots\right),
$$

где

$$
(n)_{k}=n(n-1) \ldots(n-k+1), \quad k=1,2, \ldots
$$

Для величин $K(n, 3)$ при $n \rightarrow \infty$ справедливо аналогичное разложение [40]

$$
K(n, 3) \sim(n !)^{2} e^{-3}\left(1-\frac{1}{n}-\frac{1}{2(n)_{2}}+\frac{5}{6(n)_{3}}+\ldots+\frac{b_{s}}{s !(n)_{s}}+\ldots\right),
$$

где $b_{s}=H_{s}(-1 / 2), H_{s}(t)-$ многочлены Эрмита, $s=1,2, \ldots$

Таблищы чисел $K(n, 3)$ для $n \leqslant 40$ приведены в [41]. Формулы для чисел $K(n, 4)$ найдены в [42], способ нахождения $K(n, 4)$ указан в [43].

С момента появления результатов работ [44] и [8] долгое время не было сушественного прогресса в решении сложной задачи определения асимптотики числа латинских прямоугольников $L(n, m)$. В [44] было показано, что при $n \rightarrow \infty$ и $m<(\ln n)^{3 / 2}$

$$
L(n, m)=(n !)^{m} \exp \left(-\left(\begin{array}{c}
m \\
2
\end{array}\right)\right)(1+o(1)),
$$


и высказано предположение, что эта оценка остается справедливой при $m=o\left(n^{1 / 3}\right)$. Это предположение позднее было доказано в [8]. В [5] получено усиление результатов статьи [8]:

$$
L(n, m)=(n !)^{m} \exp \left(-\left(\begin{array}{c}
m \\
2
\end{array}\right)-\frac{m^{3}}{6 n}+O\left(\max \left\{m / \sqrt{n}, m^{4} / n^{2}\right\}\right)\right)
$$

равномерно относительно $m \leqslant n / 10$.

В частности, при $n \rightarrow \infty$ и $m=o(\sqrt{n})$ справедлива формула (8).

Дальнейшим продвижением явилась работа [6] (см. выше замечание 1). В этой работе, в частности, показано, что при $n \rightarrow \infty$ и $m=o\left(n^{6 / 7}\right)$

$$
L(n, m)=(n !)^{m} n^{-m n}\left((n)_{m}\right)^{n}(1-m / n)^{-n / 2} e^{-m / 2}(1+o(1))
$$

(подробнее см. замечание 7).

Каких-либо асимптотик для числа $L_{n}=L(n, n)$ латинских квадратов порядка $n$ при $n \rightarrow \infty$ (если не считать гипотезы автора (18)) до настоящего времени не получено.

Перечисление латинских квадратов порядка $n$ было осуществлено при $n=6$ в [45], $n=7$ в [46], $n=8$ в [47], $n=9$ в [48], $n=10$ в [10]. В [10] приведены также оценки чисел $L_{n}$ при $11 \leqslant n \leqslant 16$, полученные с помощью некоторых косвенных методов, в том числе метода экстраполяции (см. выше примечание к таблице 3). В [49] приведены известные к 1975 г. точные значения $L_{n}$ при $n \leqslant 9$ и сказано, что дальнейшее определение этих значений при $n>9$ представляется крайне сложным.

Точная формула для $L_{n}$ (имеющая, по-видимому, лишь теоретический интерес) приведена в [50]:

$$
L_{n}=n ! \sum_{A \in G_{n}}(-1)^{N_{0}(A)}\left(\begin{array}{c}
\operatorname{per} A \\
n
\end{array}\right)
$$

где $G_{n}$ - множество всех $2^{n^{2}}(0,1)$-матриц размера $n \times n, N_{0}(A)$ - число нулевых элементов в матрице $A$.

Следуя [31], приведем результаты, связанные с пополнением частичного латинского квадрата.

Известно, что если в частичном латинском квадрате порядка $n$ заполнено $n$ мест, то его не всегда можно дополнить до латинского квадрата. В 1960 г. была выдвинута гипотеза [51] о том, что частичный латинский квадрат порядка $n$ с не более чем $n-1$ заполненными местами всегда может быть дополнен до латинского квадрата. Было показано [52], что можно построить латинский квадрат размера $n \times n$ с заданными $n-1$ диагональными элементами. Пусть $r$ обозначает число строк, в которых находятся $n-1$ заполненных ячеек частичного латинского квадрата порядка $n$, а $s$ - соответствующее число столбцов. В [53] установлено, что если $r \leqslant[n / 2]$ или $s \leqslant[n / 2]$, то частичный латинский квадрат можно дополнить до латинского квадрата.

Сходное условие приведено в [54]. Если в частичном латинском $n \times n$ квадрате найдется такая строка и столбец, что в этой строке и в этом столбце заполнено по крайней мере $[n / 2]$ ячеек, то такой частичный латинский квадрат тоже можно дополнить до латинского квадрата. В [55] и [56] было получено дальнейшее продвижение в доказательстве гипотезы из статьи [51]. Установлено, что частичный латинский квадрат порядка $n$ с заполненными $n-1$ ячейками можно дополнить до латинского квадрата в следующих случаях: 
(1) заполненные ячейки находятся самое болышее в $n-2 \sqrt{n}$ строках (столбцах);

(2) число различных элементов в заполненных ячейках не превосходит $n-2 \sqrt{n}$;

(3) $n$ делится на 4 и $n \geqslant 556$;

(4) $n>1111$

Тем самым упомянутая гипотеза подтверждается для всех достаточно больших значений $n$.

Множество из $r \leqslant n$ различных элементов, каждые два из которых не расположены на одной линии, называется частичной трансверсалью латинского квадрата порядка $n$. При $r=n$ это множество называется трансверсалью латинского квадрата.

Известна гипотеза Райзера (1967 г.) о том, что любой латинский квадрат нечетного порядка имеет трансверсаль. В [57] показано, что в латинском квадрате порядка $n \geqslant 7$ имеется хотя бы одна частичная трансверсаль порядка, не меньшего $(n+1) / 3$. В [58] установлено, что любой латинский $n \times n$ квадрат имеет частичную трансверсаль порядка, не меньшего $\geqslant n-\sqrt{n}$. Аналогичный результат доказан в [59]: каждый латинский $n \times n$ квадрат имеет частичную трансверсаль порядка $n-r$, где $r(r+1) \geqslant n$.

Латинским квадратам и их применениям посвящена специальная монография [60]. В книге [61] изучаются различные вопросы, связанные с теорией латинских прямоугольников и латинских квадратов (ортогональные латинские квадраты, конечные геометрии, конечные плоскости, блок-схемы, разностные множества, системы различных представителей, матрищы Адамара и др.)

\section{Список литературы}

1. Минк Х., Перманенты. Мир, Москва, 1982.

2. Брэгман Л. М., Некоторые свойства неотрицательных матриц и их перманентов. ДАН СССР (1973) 211, №1, 27-30.

3. Егорычев Г. П., Решение проблемы Ван-дер-Вардена для перманентов. Препринт, Институт физики им. Л. В. Киренского СО АН СССР, ИФСО-13М, Красноярск, 1980.

4. O'Neil P. E., Asymptotic and random matrices with row-sum and column-sum restrictions. Bull. Amer. Math. Soc. (1969) 75, №6, 1276-1282.

5. Stein C. M., Asymptotic evaluation of the number of Latin rectangles. J. Comb. Theory (1978) 25, №1, 38-49.

6. Godsil C. D., McKay B. D., Asymptotic enumeration of Latin rectangles. J. Comb. Theory (1990) 48, №1, 19-44.

7. Пазизин С. В., Об одном подходе к получению статистических оценок размера генеральной совокупности. Дискретная математика (1995) 7, №1, 123-133.

8. Yamamoto K., On the asymptotic number of Latin rectangles. Japan. J. Math. (1951) 21, 113-119.

9. Вероятность и математическая статистика. Энииклопедия. Научн. изд-во БРЭ, Москва, 1999.

10. McKay B. D., Rogoyski E., Latin squares of order 10. Electronic J. Comb. (1995), №2(3), 1-4.

11. Риордан Дж., Введение в комбинаторный анализ. ИЛ, Москва, 1963.

12. König D., Über Graphen und ihre Anwendung auf Determinanten-Theorie und Mengelehre. Math. es Termesz. Ërtesitö (1916) 34, 104-119. 
13. Van der Waerden B. L., Aufgabe 45. Jahresber. Deutsch. Math.-Vereinig. (1926) 35, 117.

14. Фаликман Д. И., Доказательство гипотезы Ван-дер-Вардена о перманенте дважды стохастической матрицы. Матем. заметки (1981) 29, №6, 931-938.

15. Minc H., Upper bounds for permanents of $(0,1)$-matrices. Bull. Amer. Math. Soc. (1963) 69, 789791.

16. Wilf H. S., On the permanent of a doubly stochastic matrix. Canad. J. Math. (1966) 18, 758-761.

17. Jurkat W. B., Ryser H. J., Matrix factorizations of determinants and permanents. J. Algebra (1966) 3, 1-27.

18. Minc H., A lower bound for permanents of $(0,1)$-matrices. Proc. Amer. Math. Soc. (1967) 18, 1128-1132.

19. Erdős P., Rényi A., On random matrices. II. Studia Sci. Math. Hungar. (1968) 3, №4, 459-464.

20. O’Neil P., Asymptotic in random (0, 1)-matrices. Proc. Amer. Math. Soc. (1970) 25, №2, 290-295.

21. Сачков В. Н., Вероятностные методы в комбинаторном анализе. Наука, Москва, 1978.

22. Everett C. J., Stein P. R., The asymptotic number of $(0,1)$-matrices with zero permanent. Discrete Math. (1973) 6, 29-34.

23. Gordon B., Motzkin T. S., Welch L., Permanents of $(0,1)$-matrices. J. Comb. Theory (1974) 17, 145-155.

24. Schrijver A., Valiant W. G., On lower bounds for permanents. Indag. Math. (1980) 42, 425-427.

25. Voorhoeve M., A lower bound for the permanents of certain $(0,1)$-matrices. Indag. Math. (1979) 41, 83-86.

26. Тимашёв А. Н., Закон больших чисел для перманентов случайных стохастических матриц. Дискретная математика (1999) 11, №3, 91-98.

27. Shevelev V. S., On "projection" of the Erdős-Rényi theorem about permanent of stochastic $(0,1)-$ matrices into the subset of stochastic $(0,1)$-matrices wich equal row sums. Тезисы докл. Третьей Всероссийской школы-коллоквиума по стохастическим методам. ТВП, Москва, 1996, с. 177178.

28. Амбросимов А. С., Тимашёв А. Н., Закон больших чисел для пропускной способности каналов без памяти со случайной переходной матрицей. Пробл. передачи информачии (1995) 31, №3, $24-34$.

29. Минеев М. П., Павлов А. И., О числе $(0,1)$-матриц с заданными суммами по строкам и столбцам. Докл. АН СССР (1976) 230, №2, 271-274.

30. Сачков В. Н., Системы различных представителей для случайных множеств. Матем. сб. (1975) 97, 397-402.

31. Носов В. А., Сачков В. Н., Тараканов В. Е., Комбинаторный анализ (Матричные проблемы, теория выбора). Итоги науки и техники, сер. Теория вероятностей. Математическая статистика. Теоретическая кибернетика. ВИНИТИ, Москва, 1981, т. 18, с. 53-93.

32. Рыбников К. А., Введение в комбинаторный анализ. МГУ, Москва, 1972.

33. Сачков В. Н., Комбинаторные методы дискретной математики. Наука, Москва, 1977.

34. Райзер Г. Дж., Комбинаторная математика. Мир, Москва, 1966.

35. Сачков В. Н., Комбинаторные свойства неотрицательных матриц. Пробл. киберн. (1978) 26, $37-50$.

36. Сачков В. Н., Тараканов В. Е., Комбинаторика неотричательных матрич. ТВП, Москва, 2000.

37. Королюк В. С., Боровских Ю. В., Случайные перманенты. Ин-т математики АН Украины, Киев, 1993. 
38. Touchard J., Sur un problème de permutations. C. R. Acad. Sci. Paris (1934) 198, 631-633.

39. Riordan J., Three-line Latin rectangles. Amer. Math. Monthly (1944) 51, 450-452.

40. Yamamoto K., An asymptotic series for the number of three-line Latin rectangles. J. Math. Soc. Japan (1949) 1, 226-241.

41. Gergely E., A simple method for constructing doubly diagonalized Latin squares. J. Comb. Theory (1974) 16, №2, 266-272.

42. Добровольский М. А., О четырехстрочных латинских прямоугольниках. Материалы межвузовской научной конф. пед. ин-тов чентр. зоны. Тула, 1968, с. 72-75.

43. Light F. W., Enumeration of truncated Latin rectangles. Fibonacci Quart. (1979) 17, №1, 34-36.

44. Erdős P., Kaplansky I., The asymptotic number of Latin rectangles. Amer. J. Math. (1946) 68, 230-236.

45. Fisher R., Yates F., The $6 \times 6$ Latin squares. Proc. Cambridge Philos. Soc. (1934) 30, 492--507.

46. Norton H., The $7 \times 7$ squares. Ann. Eugenics (1939) 9, 269-307.

47. Wells B., The number of Latin squares of order 8. J. Comb. Theory (1967) 3, 98-99.

48. Bammel S., Rothstein J., The number of $9 \times 9$ Latin squares. Discrete Math. (1975) 11, 93-95.

49. Alter R., How many Latin squares are there? Amer. Math. Monthly (1975) 82, 632-634.

50. Jiayu Shao, Wandi Wei, A formula for the number of Latin squares. Discrete Math. (1992) 110, 239-246.

51. Evans T., Embedding incomplete Latin squares. Amer. Math. Monthly (1960) 67, №10, 958-961.

52. Marica J., Schönheim J., Incomplete diagonals of Latin squares. Can. Math. Bull. (1969) 12, №2, 235.

53. Lindner C. C., On completing Latin rectangles. Can. Math. Bull. (1970) 13, №1, 65-68.

54. Dacic R., On the completion of incomplete Latin squares. Publ. Inst. Math., Beograd (1978) 23, 75-80.

55. Häggkvist R., A partial solution of the Evans conjecture for Latin squares. Dep. Math. Univ. Umea, 1976.

56. Häggkvist R., A solution of the Evans conjecture for Latin squares of large size. Combinatorics (1978) 1, 495-513.

57. Koksma K. K., A lower bound for the order of a partial transversal in a Latin square. J. Comb. Theory (1969) 7, №1, 94-95.

58. Woolbright $\mathrm{D}$. E., An $n \times n$ Latin square has a transversal with at least $n-\sqrt{n}$ distinct symbols. $J$. Comb. Theory (1978) 24, №2, 235-237.

59. Brouwer A. E., de Vries A. J., Wieringa R. M. A., A lower bound for the length of partial transversals in a Latin square. Nieuw Arch. Wiskd., III, Ser. 26 (1978) 330-332.

60. Denes J., Keedwell A. D., Latin squares and their applications. Akademiai Kiado, Budapest, 1974.

61. Холл М., Комбинаторика. Мир, Москва, 1970. 